\title{
Measurements on irradiated L1 sensor prototypes for the D0 Run Ilb silicon detector project
}

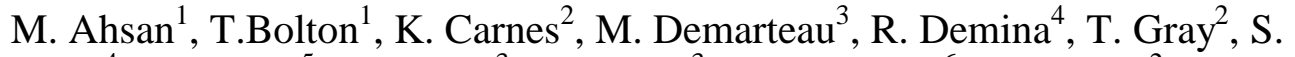 \\ Korjenevski ${ }^{4}$, F. Lehner ${ }^{5}$, R. Lipton ${ }^{3}$, H.S. Mao ${ }^{3}$, R. McCarthy ${ }^{6}$, A. Rankin ${ }^{2}$, M. Shamin ${ }^{1}$, \\ R.P. Smith ${ }^{3}$ \\ 1 Kansas State University, HEP group, Manhatten, KS, USA \\ 2 Kansas State University, James R. Mcdonald Laboratory, Manhatten, KS, USA \\ 3 Fermi National Accelerator Laboratory, Batavia, IL, USA \\ 4 University of Rochester, NY, USA \\ 5 Physics Institute of University of Zurich, Switzerland \\ 6 State University of New York at Stony Brook, USA
}

\begin{abstract}
We report on irradiation studies of Hamamatsu prototype silicon microstrip detectors for layer 1 of the D0 upgrade project for Run IIb. The irradiation was carried out with 10 $\mathrm{MeV}$ protons up to proton fluence of $10^{14} \mathrm{p} / \mathrm{cm}^{2}$ at the J.R. Macdonald Laboratory, Manhatten, KS. The flux calibration was carefully checked using different dose normalization techniques. The results based on the obtained sensor leakage currents after irradiation show that the NIEL scaling hypothesis for low energy protons has to be applied with great care. We observe $30-40 \%$ less radiation damage in silicon for $10 \mathrm{MeV}$ proton exposure than is expected from the predicted NIEL scaling.
\end{abstract}

\section{Introduction}

Over the course of Run IIb at the Tevatron, the silicon detectors in the inner layers will be exposed to a harsh radiation environment. The expected fluence ${ }^{1}$ in layer 0 and layer 1 silicon sensors (hereafter called L0 and L1) including a safety factor of 1.5 will be $1.25 \cdot 10^{13} 1 \mathrm{MeV} \mathrm{n} / \mathrm{cm}^{2} / \mathrm{fb}^{-1}$ and $4 \cdot 10^{12} 1 \mathrm{MeV} \mathrm{n} / \mathrm{cm}^{2} / \mathrm{fb}^{-1}$ respectively. L1 prototype silicon sensors from Hamamatsu (HPK) based on our L1 sensor specifications ${ }^{2}$ have arrived. The L1 sensors are standard p+n silicon sensors with a thickness of $320 \mu \mathrm{m}$ and have the typical HPK single guard ring design. In order to study the radiation hardness of some of these delivered L1 silicon sensor prototypes, an irradiation study was carried out. Three HPK L1 silicon sensor prototypes were irradiated in $10 \mathrm{MeV}$ protons beams up to a final fluence of $9.35 \times 10^{13} 10 \mathrm{MeV} \mathrm{p} / \mathrm{cm}^{2}$.

\footnotetext{
${ }^{1}$ F. Lehner, New expectations for depletion voltages and leakage currents for the Run IIb, D0-note 3959

${ }^{2}$ http://www.physik.unizh.ch/ lehnerf/dzero/specs/specs.html
} 
Furthermore, an irradiation series with single guarded planar diodes was performed at five different fluence points up to an accumulated fluence of $1.32 \times 10^{14} 10 \mathrm{MeV} \mathrm{p} / \mathrm{cm}^{2}$. The diodes were part of test structures that were delivered by the supplier. The irradiation of the L1 silicon sensors was performed in up to seven successive steps, until the total dose was accumulated, while for the diodes one different fluence point for each diode was chosen. After each irradiation the detectors were properly annealed and the depletion voltage and leakage currents were measured.

In our irradiation study we have used $10 \mathrm{MeV}$ protons due to the availability and easy access to the Tandem Van de Graaff accelerator at Kansas State University. Most of the results on silicon detector irradiation tests in the literature however, come either from low energy neutrons or charged hadrons at rather high energies. Nevertheless, we are aware of two other irradiation studies of silicon detectors with low energy protons. One of the studies $^{3}$ was done by the ROSE collaboration and has used 7-10 MeV protons at a similar accelerator at the University of Montreal. Their results are used as comparisons. In the following we will refer to this irradiation study as the "Montreal" study. The other study ${ }^{4}$ was carried out at INFN Legnaro laboratory using 16, 21 and $27 \mathrm{MeV}$ protons.

\section{Experimental Setup}

The irradiation was carried out at the 7MV Tandem van de Graaf accelerator of the J.R. Macdonald laboratory at $\mathrm{KSU}^{5}$. The beam energy of the protons was set to $10 \mathrm{MeV}$. The irradiation dose was carefully measured using charge collected by a Faraday cup, which was read out by a charge integrator. An independent verification and cross check of the Faraday cup dose determination was done using activation measurements of 1.5 mil thick $\mathrm{Cu}$ foils confirming the Faraday cup measurements on a 15\% level. The activation analysis carried out at KSU was double checked at FNAL. Exact details of the beam properties, the experimental setup, the Faraday cup calibrations and the flux cross checks by two independent activation measurement analysis are summarized in the accompanying note "Flux normalization for $10 \mathrm{MeV}$ protons used in RunIIb Radiation Tests"

The sensors and diodes were mounted on aluminium holders having a large enough cutout and placed inside an evacuated target chamber. A photograph showing the diodes on one half moon shaped teststructure is visible in Figure 1.

\footnotetext{
${ }^{3}$ D. Bechevet et al. "Results on irradiation tests on planar silicon detectors with 7-10 MeV protons", NIM A 479 (2002) 487

${ }^{4} \mathrm{~J}$. Wyss et al. "Observation of an energy dependance of the radiation damage on standard and oxygenated silicon diodes by 16, 21, and $27 \mathrm{MeV}$ protons", NIM A 457 (2001) 595

${ }^{5} \mathrm{http}: / / \mathrm{www}$. phys.ksu.edu/area/jrm

${ }^{6} \mathrm{~T}$. Bolton et al. "Flux Normalization for $10 \mathrm{MeV}$ protons used in Run IIb Radiation Tests"
} 


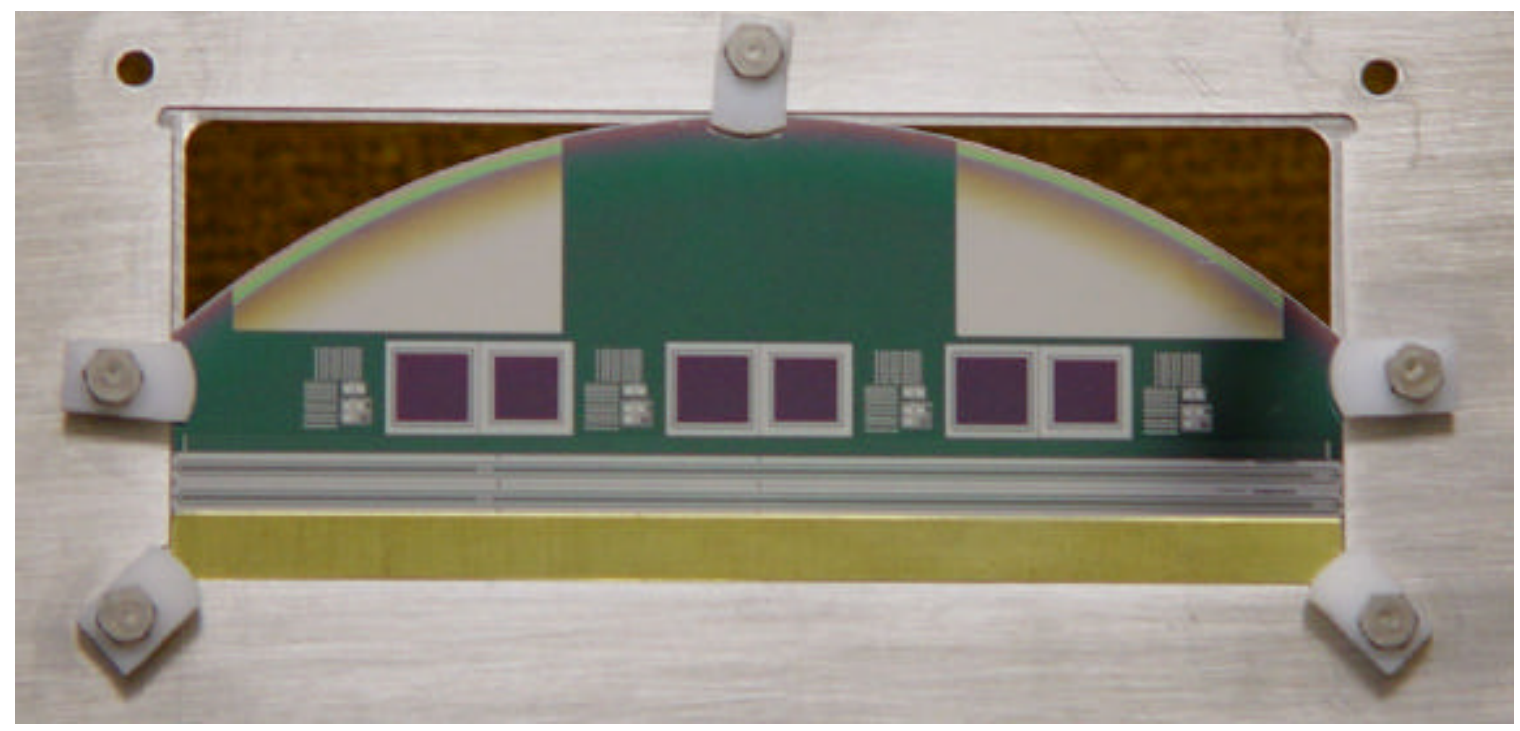

Figure 1: Photograph of the square diodes on the half moon test structures in the Aluminium frame.

The annealing of the L1 sensors after each irradiation step was performed at $60^{\circ} \mathrm{C}$ for 80 minutes as suggested by M. Moll et al ${ }^{7}$. Immediately after the annealing the I-V and C-V characterizations was performed on a $1^{\circ} \mathrm{C}$ cold probe station chuck with standard equipment ${ }^{8}$.

\section{Radiation hardness of $10 \mathrm{MeV}$ protons}

The application of the so-called Non Ionizing Energy Loss (NIEL) scaling is based on the hypothesis that the induced displacement damage in silicon bulk material scales linearly with non-ionizing energy transfer to the lattice. It is then common to normalize any particle fluence $\Phi$ to the equivalent $1 \mathrm{MeV}$ n fluence

$\Phi(1 \mathrm{MeV}$ eq $\mathrm{n})=\kappa \cdot \Phi$

with $\kappa$ being the hardness factor. Theoretical values for the damage displacement function $\mathrm{D}_{\mathrm{i}}(\mathrm{E})$ for particle $\mathrm{i}$ and energy $\mathrm{E}$ normalized to the $1 \mathrm{MeV}$ neutron displacement functions are tabulated ${ }^{9}$. For $10 \mathrm{MeV}$ protons the theoretical expected hardness factor $\mathrm{\kappa}$ is 3.87. It has to be noted however, that due to the finite silicon thickness of $320 \mu \mathrm{m}$ for our sensors, energy loss for $10 \mathrm{MeV}$ protons occurs in the silicon. The theoretical range of 10 $\mathrm{MeV}$ protons in silicon corresponds to $700 \mu \mathrm{m}$, so that the theoretical hardness factor for finite thickness silicon is in fact higher, since the displacement function $\mathrm{D}(\mathrm{E})$ for protons

\footnotetext{
${ }^{7}$ M. Moll et al., "Leakage currents of hadron irradiated silicon detectors - material dependence", NIM A 426 (1999) 87

${ }^{8}$ Keithley 237 source measurement units and HP 4284 LCR meter

${ }^{9}$ G.P. Summers et al., Damage correlations in semiconductors exposed to gamma, electron and proton radiation, IEEE Trans. Nucl. Sci. NS-40,6 (1993) 1372
} 
rises towards lower energies. The Montreal paper $^{3}$ cites a calculation which was performed for $295 \mu \mathrm{m}$ thick silicon yielding a corrected hardness factor of 4.5 for 10 $\mathrm{MeV}$ protons.

\section{Experimental Results on Leakage Currents}

The measured leakage current data for three irradiated L1 sensors at various $10 \mathrm{MeV}$ proton fluences is presented below. All leakage current measurements at any fluence point were performed after the aforementioned annealing procedure, so that most of the short term annealing in the silicon bulk material has then taken place. The leakage currents, which are shown in the graph were measured at a temperature of $1^{\circ} \mathrm{C}$.

Sensor HPK-L1-11 as seen in

Figure 2 exhibits a sudden increase in leakage currents at a bias voltage of around $750 \mathrm{~V}$ during the I-V measurement at a fluence point of $5.23 \cdot 10^{11} \mathrm{p} / \mathrm{cm}^{2}$. This breakdown however, is not reproduced towards higher fluences. It is therefore not very conclusive to attribute its behavior to a junction breakdown. The second L1 sensor, HPK-L1-12, is plotted in Figure 3. The sensor showed no junction breakdown up to the specified voltage of $700 \mathrm{~V}$.

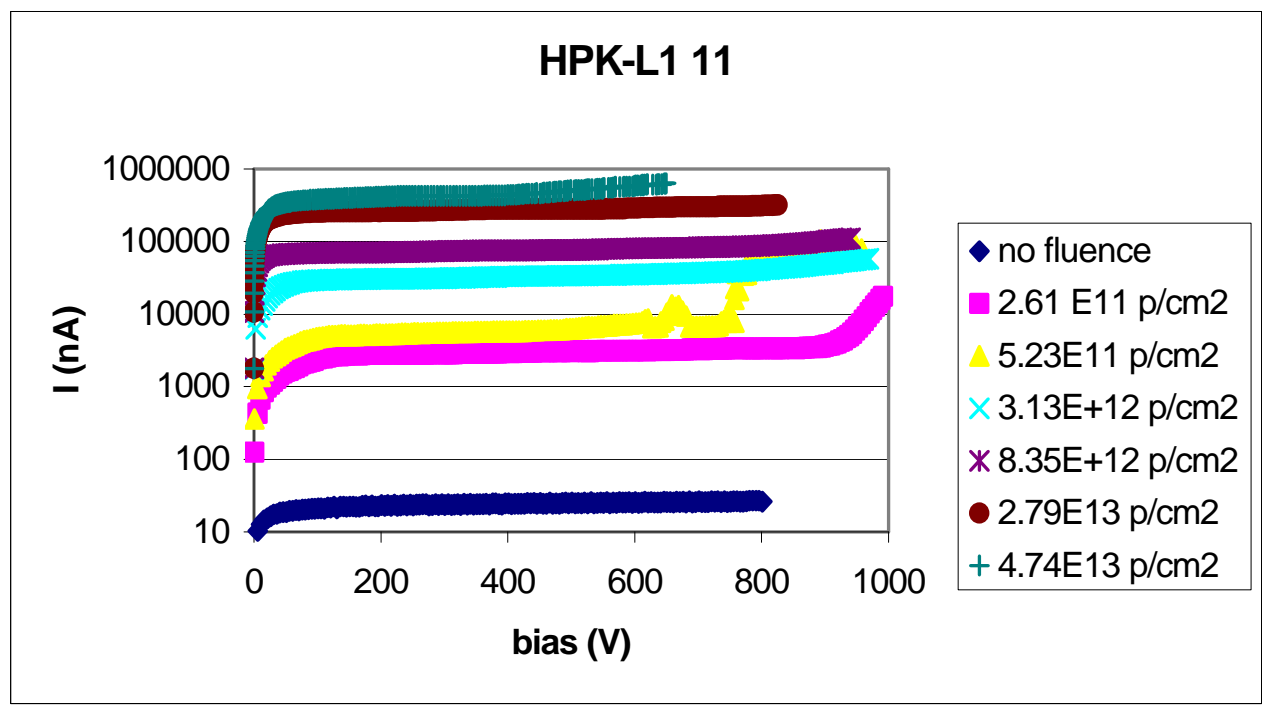

Figure 2: Leakage current measured at 1C for HPK-L1-11 for different $10 \mathrm{MeV}$ proton fluences 


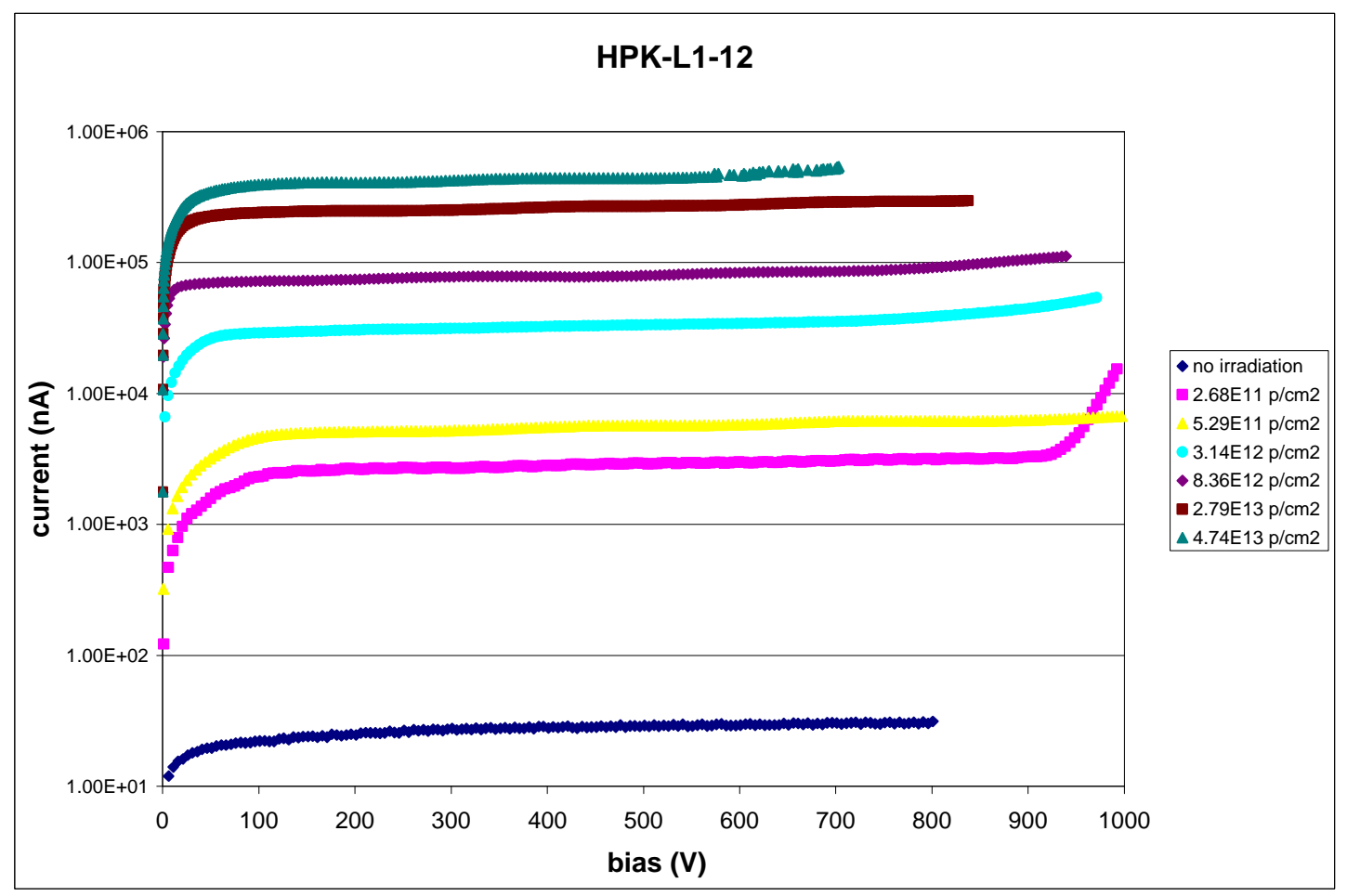

Figure 3: Leakage current measured at $1 \mathrm{C}$ for HPK-L1-12 for different $10 \mathrm{MeV}$ proton fluences.

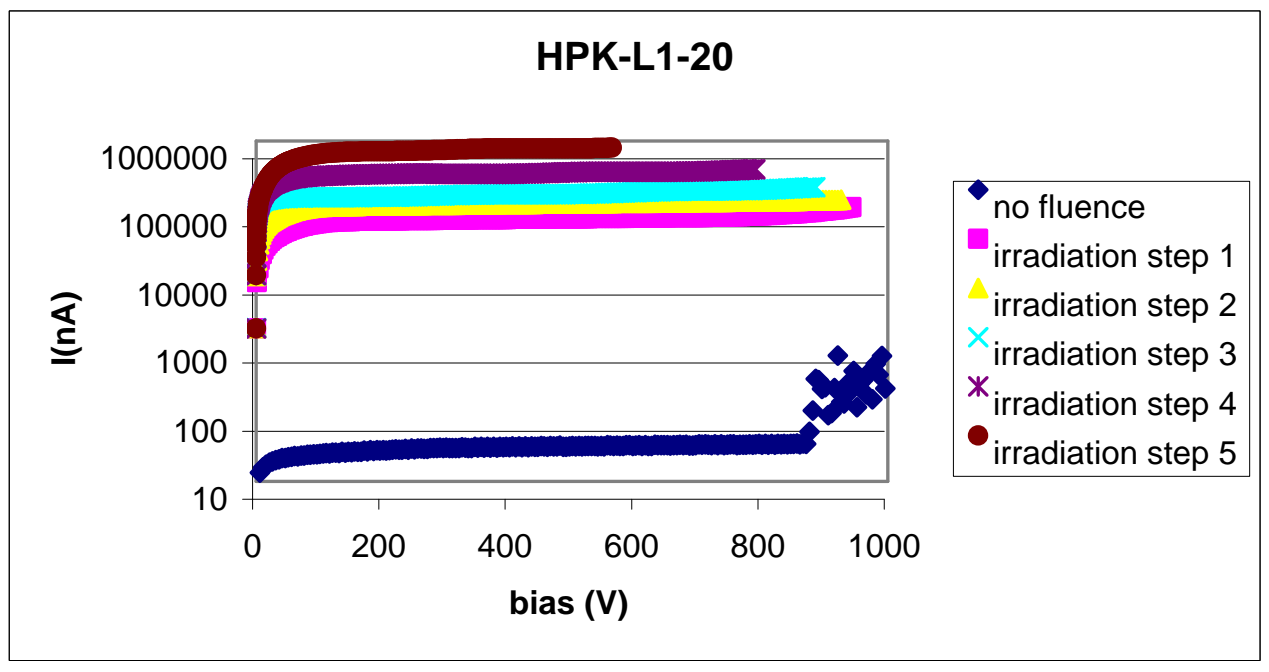

Figure 4: Leakage currents measured at 1C for HPK-L1-20 at various fluence points.

During the irradiation session, the third sensor, HPK-L1-20, was mounted on the back of another silicon sensor. The correct fluence numbers relevant for this particular detector can be obtained if the energy losses in the upstream sensor are taken into account. Effectively, the exposure on this downstream sensor corresponds roughly to a $7 \mathrm{MeV}$ proton irradiation. Although sensor HPK-L1-20 showed an onset of a junction breakdown before irradiation at around $900 \mathrm{~V}$, the junction behavior turned out to be stable during 
irradiation. Unfortunately, the I-V characterization on HPK-L1-20 was only done up to $600 \mathrm{~V}$ for the highest fluence point.

The sensor leakage current values have then been normalized from the measured temperature $\mathrm{T}_{\mathrm{m}}$ to the conventional reference temperature of $\mathrm{T}=20^{\circ} \mathrm{C}$ according to the relation

$\mathrm{I}(\mathrm{T})=\mathrm{I}\left(\mathrm{T}_{\mathrm{m}}\right) \cdot\left(\mathrm{T} / \mathrm{T}_{\mathrm{m}}\right)^{2} \cdot \exp \left(-\mathrm{E}_{\mathrm{g}} \cdot\left\{1 / \mathrm{T}-1 / \mathrm{T}_{\mathrm{m}}\right\} / 2 \mathrm{k}\right)$.

Here, $\mathrm{E}_{\mathrm{g}}$ is the band gap energy in silicon at room temperature taken to be $1.12 \mathrm{eV}$ and $\mathrm{k}$ is the Boltzman constant. The above current-temperature relation holds true if the current is caused by generation current in the bulk material, which is certainly fulfilled after irradiation. In order to double-check our assumed silicon temperature during probing on the cold chuck $\left(1^{\circ} \mathrm{C}\right)$, the chuck temperature has been increased and leakage currents from an irradiated sensor were recorded. The results of the check are shown in Figure 5. The measured leakage currents agree well with the expected current Temperature relation giving confidence in the cold chuck setup.

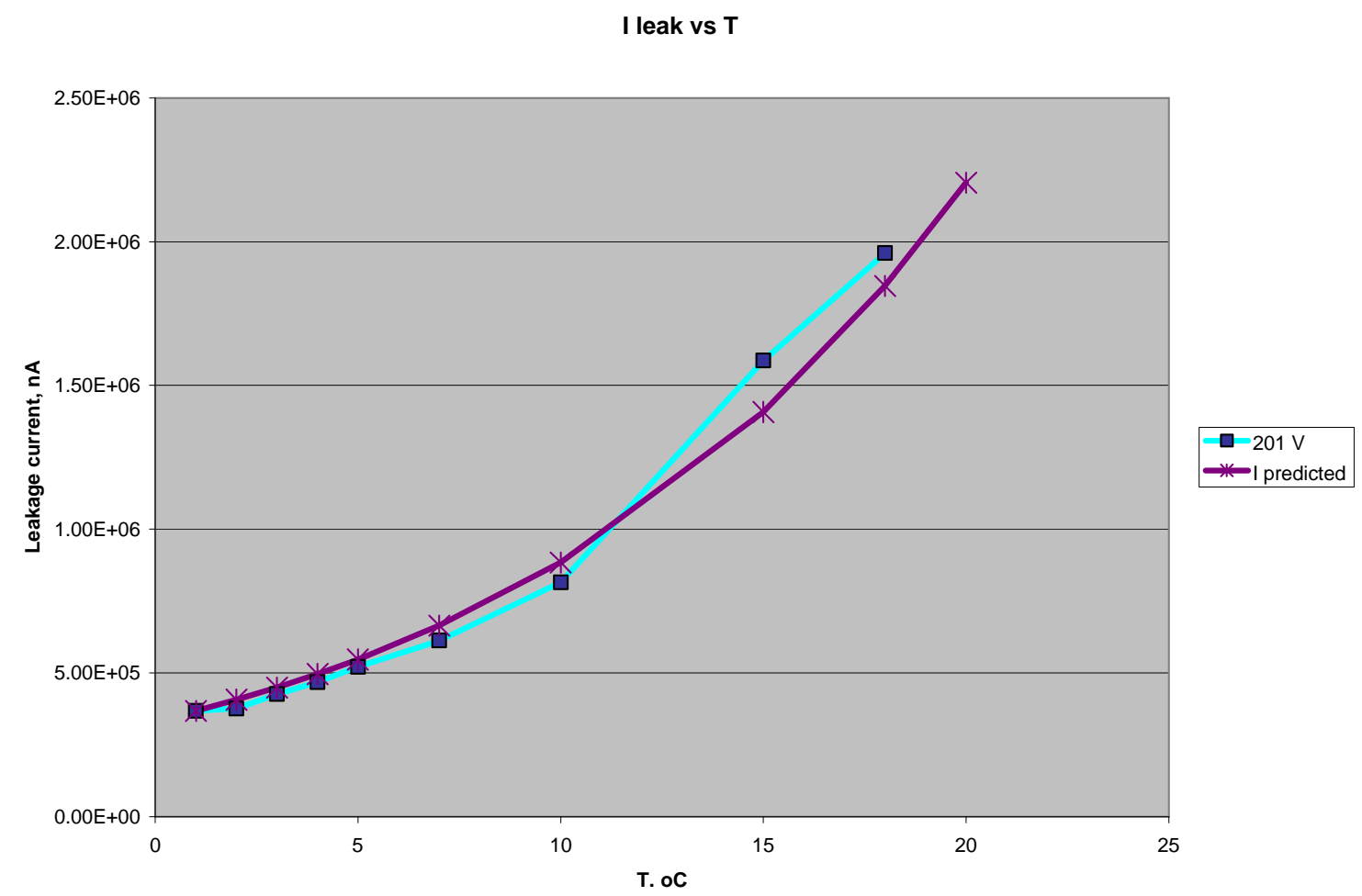

Figure 5 Verification of the silicon temperature during probing on the cold chuck. 
It is well known ${ }^{7}$ that the leakage currents normalized to the sensor volume scale linearly with the fluence. The proportionality factor between scaled leakage currents and fluence for a given particle type is called the current related damage constant $\alpha$. As is the custom in irradiation studies we have normalized our measured leakage current densities to $\mathrm{T}=20^{\circ} \mathrm{C}$ using the aforementioned relation.

In Figure 6, the leakage current densities normalized to $20^{\circ} \mathrm{C}$ are shown as function of 10 $\mathrm{MeV}$ proton fluences. We have compared the HPK-L1 and HPK-L2 data with our testdiode data and with data from the ROSE-Collaboration in their Montreal study ${ }^{3}$. The agreement among the various data sets is good. We obtain a value for $\alpha$ for $10 \mathrm{MeV} p$ of $11.6 \cdot 10^{-17} \mathrm{~A} / \mathrm{cm}$ excluding the Montreal data. Moreover, the slope does not change significantly if the Montreal data are included in the fit. The comparison to the Montreal data set is justified, since the data were obtained at the same proton energy. Although the annealing procedure in the ROSE study was carried out at higher temperatures $\left(80^{\circ} \mathrm{C}\right)$ the annealing time was shorter ( 4 min only). Both annealing procedures give in fact very similar results for the curing of the short-term damage, so that the data sets can be treated on the same footing.

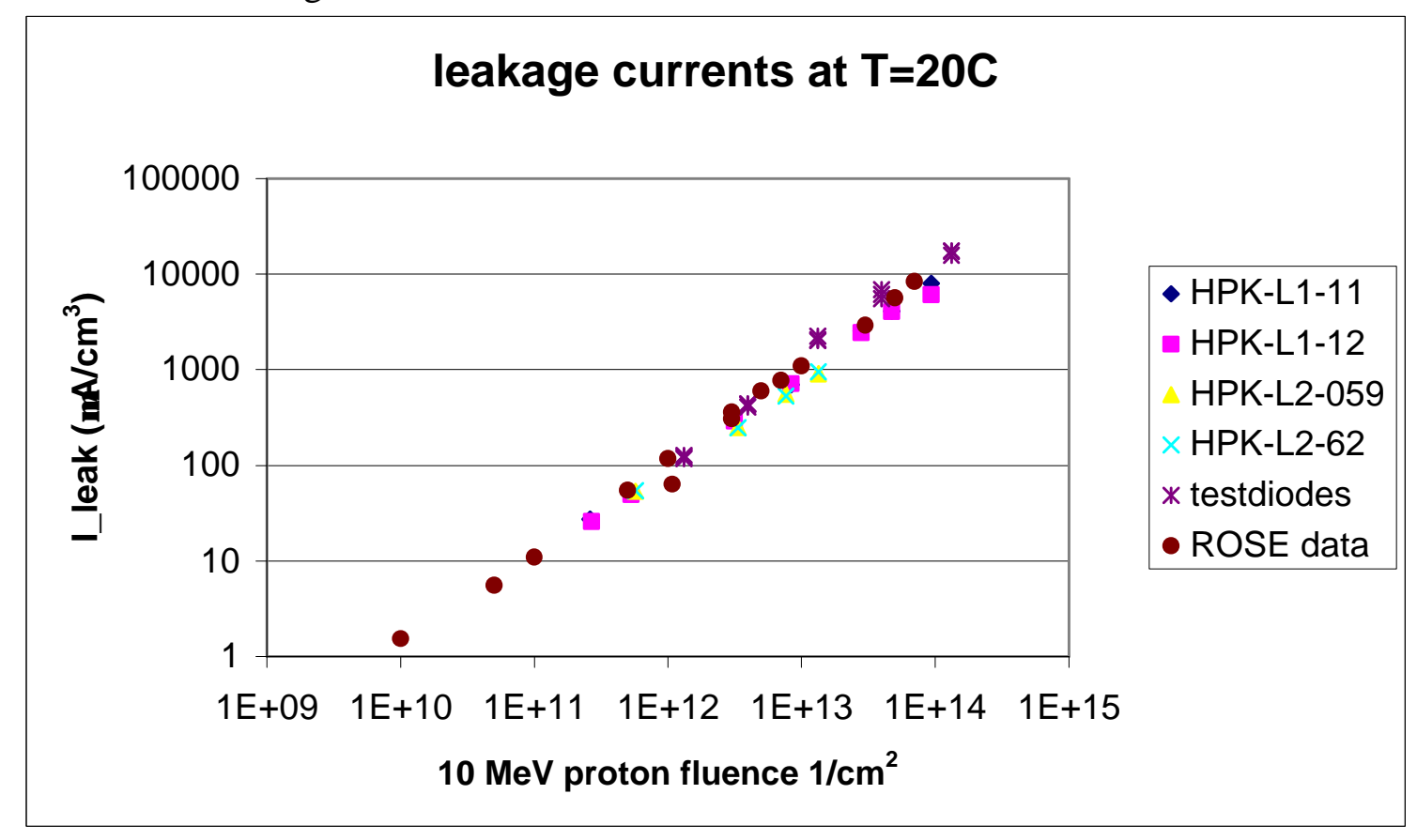

Figure 6: Leakage currents $\left(\mu \mathrm{A} / \mathrm{cm}^{3}\right)$ normalized to the detector volumes as function of the exposed 10 MeV proton fluence. The currents are scaled to T=20C. Shown are data on HPK-L1 and HPK-L2, as well as our testdiode data and the datapoints from the Montreal (ROSE-Collaboration) study. 


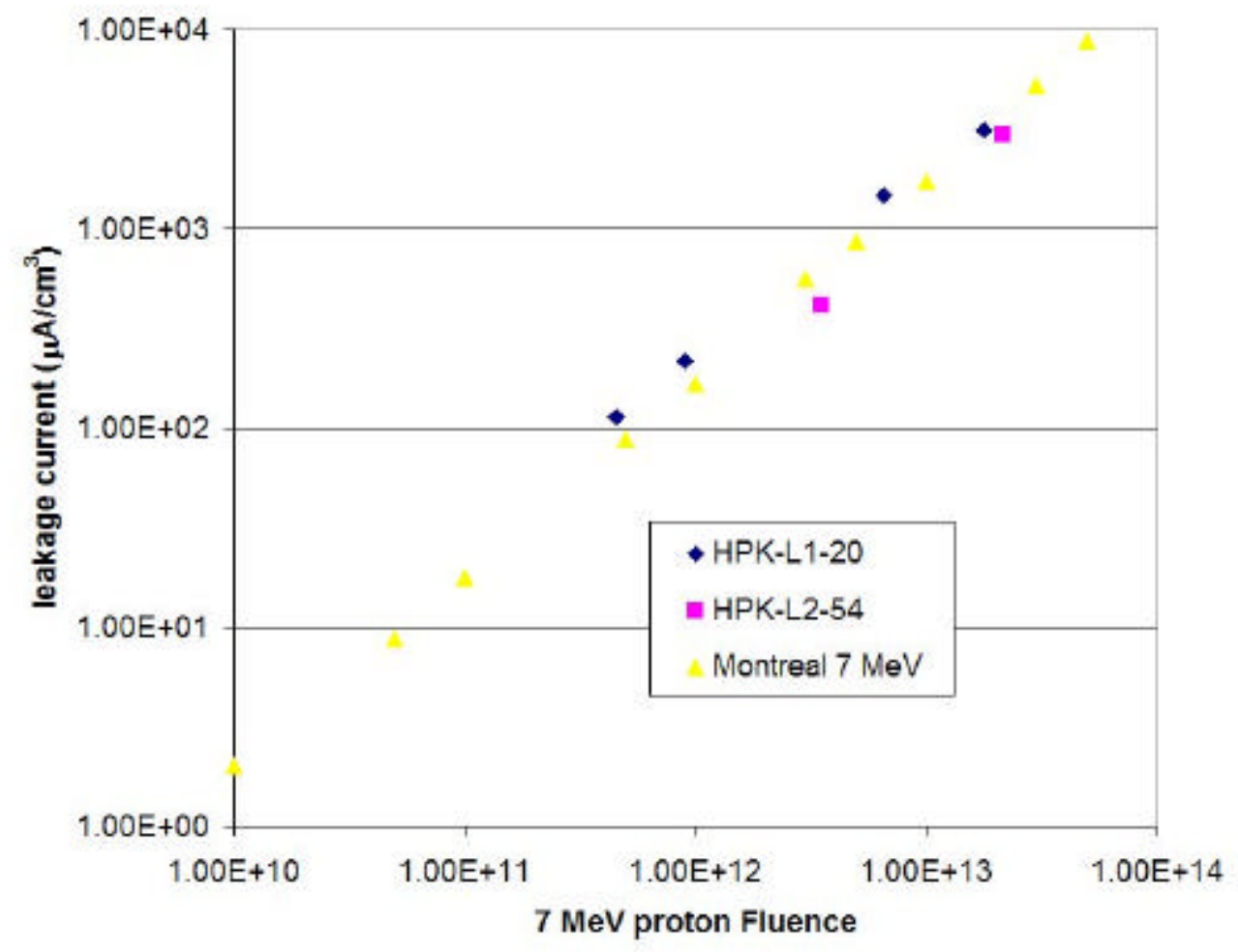

Figure 7: The leakage currents $\left(\mu \mathrm{A} / \mathrm{cm}^{3}\right)$ normalized to $20 \mathrm{C}$ as function of fluence for $7 \mathrm{MeV}$ protons. Our data on HPK-L1 and HPK-L2-54 are compared to a data set from the Montreal study.

Figure 7 shows the leakage current results for two HPK sensors, which have been irradiated in a downstream position, i.e. on the back of another sensor. By taking the energy loss into account we find reasonable agreement to the corresponding Montreal data set. A linear fit to the six available data points of our HPK sensors yields a current related damage constant $\alpha$ for the assumed $7 \mathrm{MeV}$ protons of $18.3 \cdot 10^{-17} \mathrm{~A} / \mathrm{cm}$, i.e. almost a factor of two larger than for the $10 \mathrm{MeV}$ proton irradiation case. This result compares quite well to the $7 \mathrm{MeV}$ Montreal result ${ }^{3}$ of $17.2 \cdot 10^{-17} \mathrm{~A} / \mathrm{cm}$.

It is now possible to compare the hardness factor of the proton beam as derived via the leakage current measurement to the expected hardness for $10 \mathrm{MeV}$ protons based on the NIEL scaling hypothesis. The average $\alpha$ value for $1 \mathrm{MeV}$ neutrons is taken to be $4.56 \cdot 10^{-}$ ${ }^{17} \mathrm{~A} / \mathrm{cm}$ as suggested in reference ${ }^{10}$. The ratio of the measured $10 \mathrm{MeV}$ proton alpha value to the average $1 \mathrm{MeV}$ neutron value is then the experimentally observed hardness of our beam. Hence, we find $\kappa=\alpha(10 \mathrm{MeV} p) / \alpha(1 \mathrm{MeV} \mathrm{n})=2.54$ for $10 \mathrm{MeV}$ protons. The ratio of 2.54 is significantly smaller than the tabulated hardness factor ${ }^{9}$ for $10 \mathrm{MeV}$ protons of 3.87 , i.e. we observe roughly $30-40 \%$ less radiation induced damage than expected by a simple application of the NIEL hypothesis. By taking the calculated energy loss of $10 \mathrm{MeV}$ p in finite thickness silicon (hardness of 4.5 for $290 \mu \mathrm{m} \mathrm{Si}$ ) into account,

\footnotetext{
${ }^{10}$ E. Fretwurst et al., Proceedings of the Defect Engineering of Advanced Semiconductor Devices Workshop, pp. 39-49, Santorini, Greece, 21-22. April, 1999
} 
the discrepancy to this expected hardness factor becomes even worse. It is important to note that the same conclusions have been reached by the Montreal study and -as far as we know- it is now for the first time that their findings on low energy proton damages are confirmed. A similar conclusion is reached for the HPK sensors, which have been irradiated at a degraded energy of $7 \mathrm{MeV} \mathrm{p}$. Here, the hardness of the $7 \mathrm{MeV}$ proton beam is found as $\kappa=\alpha(7 \mathrm{MeV} \mathrm{p}) / \alpha(1 \mathrm{MeV} \mathrm{n})=4.0$. This is again smaller than 5.14, which is the anticipated theoretical number from the NIEL scaling.

Our observations confirm the bulk damage related leakage currents on our sensors and planar diodes exhibit a linear scaling with proton fluences as expected. We did not observe any clear evidence for an early junction breakdown up to $700 \mathrm{~V}$ or any abnormally high currents on the HPK sensors. The temperature scaling of the bulk currents behaved as expected. The induced bulk damage in silicon based on volumetric leakage current measurements agree quite well with existing measurement from the Montreal study. Thus, we do not see any additional current generating effects due to surface damages or imperfect guard ring designs. The radiation induced bulk damage for $10 \mathrm{MeV}$ protons however, is $30-40 \%$ smaller than anticipated by NIEL scaling.

Finally, we note that by applying our $10 \mathrm{MeV}$ proton hardness factor of 2.54 , the highest proton fluences to which the sensors were exposed in our study may be converted into a 1 $\mathrm{MeV}$ equivalent neutron fluence of $2.4 \cdot 10^{14} 1 / \mathrm{cm}^{2}$. This is in fact higher than the expected radiation load (including a safety factor of 1.5 ) for L0 sensors in Run IIb.

\section{Depletion Voltage Measurements}

Compared to Run IIa silicon sensors, we have requested rather low resistivity wafers from HPK, so that the sensors are able to better withstand the high radiation environment of Run IIb. Figure 8 shows a typical bulk capacitance versus bias voltage behavior of the HPK-L1 detectors before irradiation. It is quite easy and straightforward to determine the depletion voltage from such curves. We have defined the sensor depletion voltage as the voltage point where two straight lines intersect - in this case, this corresponds to $117 \mathrm{~V}$. 
$1 \backslash C^{\wedge} 2$ vs Voltage

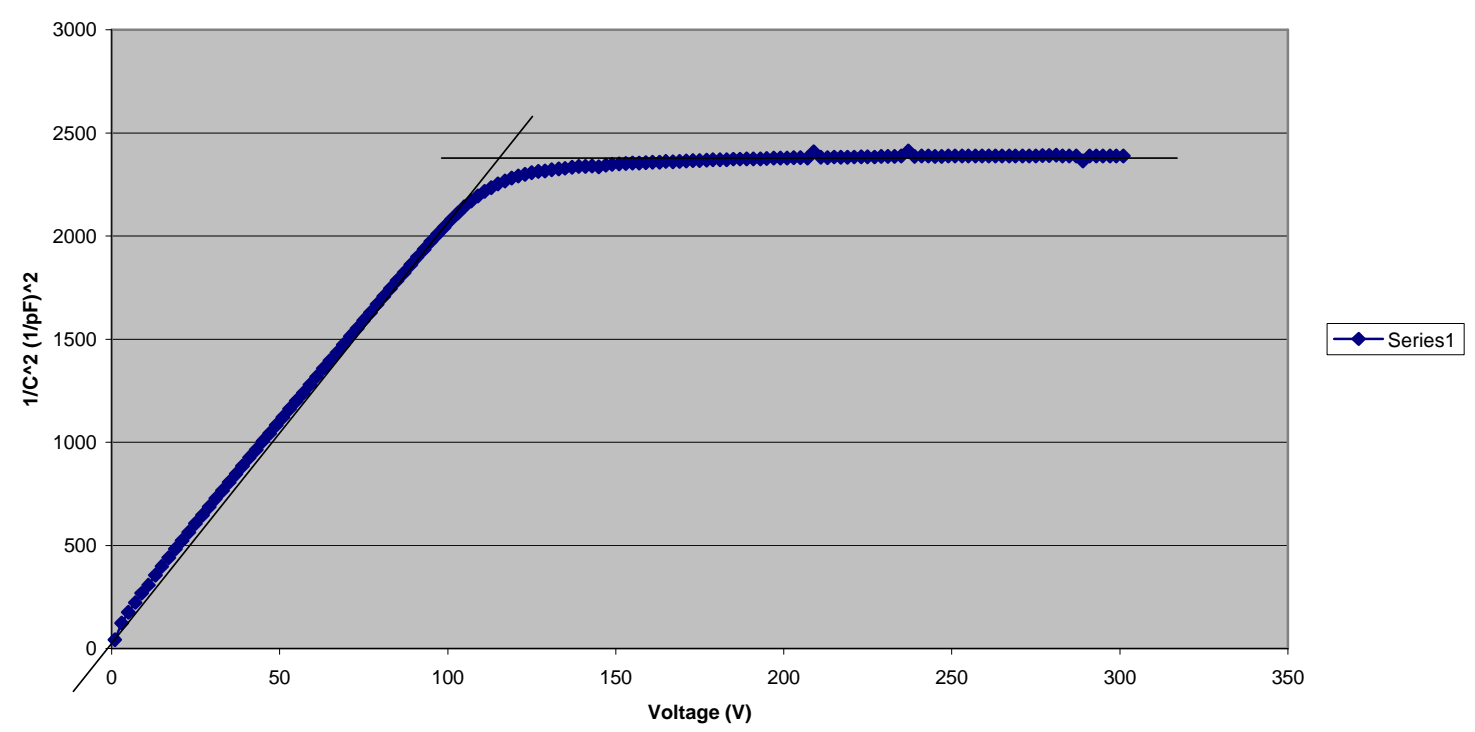

Figure 8: The plot shows the $1 / C^{2}$ versus bias dependence for one HPK-L1. The depletion voltage is determined to be $117 \mathrm{~V}$.

After each irradiation session the detectors were allowed to anneal at $60^{\circ} \mathrm{C}$ for 80 minutes and an electric testing of these sensors was done at $1^{\circ} \mathrm{C}$ temperature on the probe station chuck, in order to minimize the influence of the large leakage currents. The $\mathrm{C}-\mathrm{V}$ graphs in Figure 9 show now the $1 / \mathrm{C}^{2}$-behavior of an irradiated HPK sensor at various fluences. We still interpret the depletion voltage as being the point of two intersecting straight lines, although the value is much more sensitive to the choice of points used in the two lines. That is why we have to treat the depletion voltage results after irradiation with a much greater care than the leakage current results. An error assignment of at least 30$50 \%$ on the depletion voltage results is therefore appropriate.

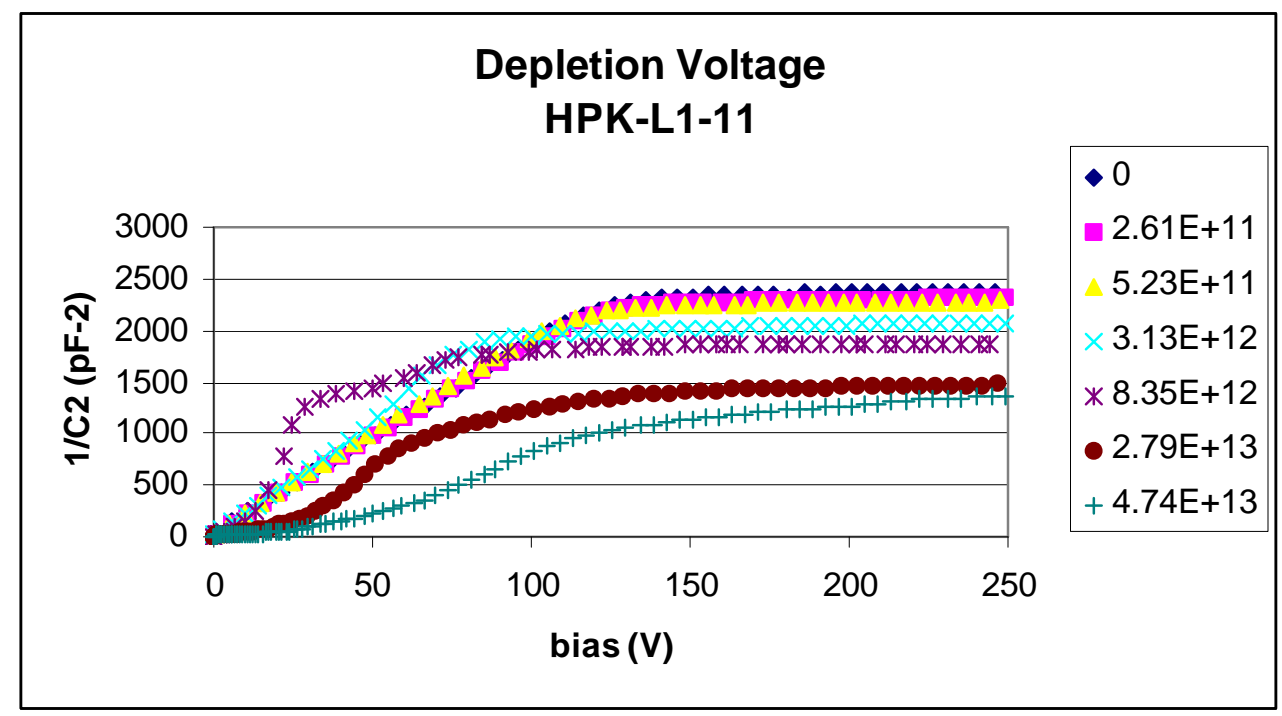


Figure 9: The plot shows the 1/C ${ }^{2}$ versus bias dependence of the HPK L1-11 sensor at several fluence

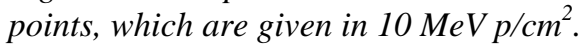

The evolution of the depletion voltage towards higher proton fluences for the irradiated HPK diodes on the test structures is presented in Figure 10. The plot also contains results from the Montreal study for comparison. In the Montreal study as well as and in ours, a diode set from the same material and with almost identical properties was employed and a particular diode from this set was exposed only once to a certain fluence point and then annealed and characterized. This procedure simply avoids further annealing effects in future irradiation steps, which could come from previous exposures. Recall that the Montreal study used a much shorter annealing time at higher temperature than our study. The diode data in Figure 10 agree quite well with each other. The Montreal data populate more the low fluence regions before and shortly after type inversion, while our data go up to the highest proton fluence point at $1.32 \cdot 10^{14} \mathrm{p} / \mathrm{cm}^{2}$.

Figure 10 also shows a simple parameterization for the effective doping concentration $\mathrm{N}_{\text {eff }}$ of the diodes as function of the proton fluence. The parameterization assumes a complete donor removal in proton irradiation as observed by ${ }^{11}$. We adopted this parameterization $\mathrm{N}_{\mathrm{eff}}=\mathrm{N}_{\mathrm{eff}, 0} \cdot \exp (-\mathrm{c} \cdot \Phi)-\mathrm{b} \cdot \Phi$ and have used for this description of the test diode data the following parameters: $c=1.13 \cdot 10^{-13} \mathrm{~cm}^{-2}$ and $b=3.4 \cdot 10^{-2} \mathrm{~cm}^{-1}$. These values were used for the $10 \mathrm{MeV}$ proton data in the Montreal paper as well. As it shown in Figure 10, the parameterization describes the observed depletion voltage changes rather well.

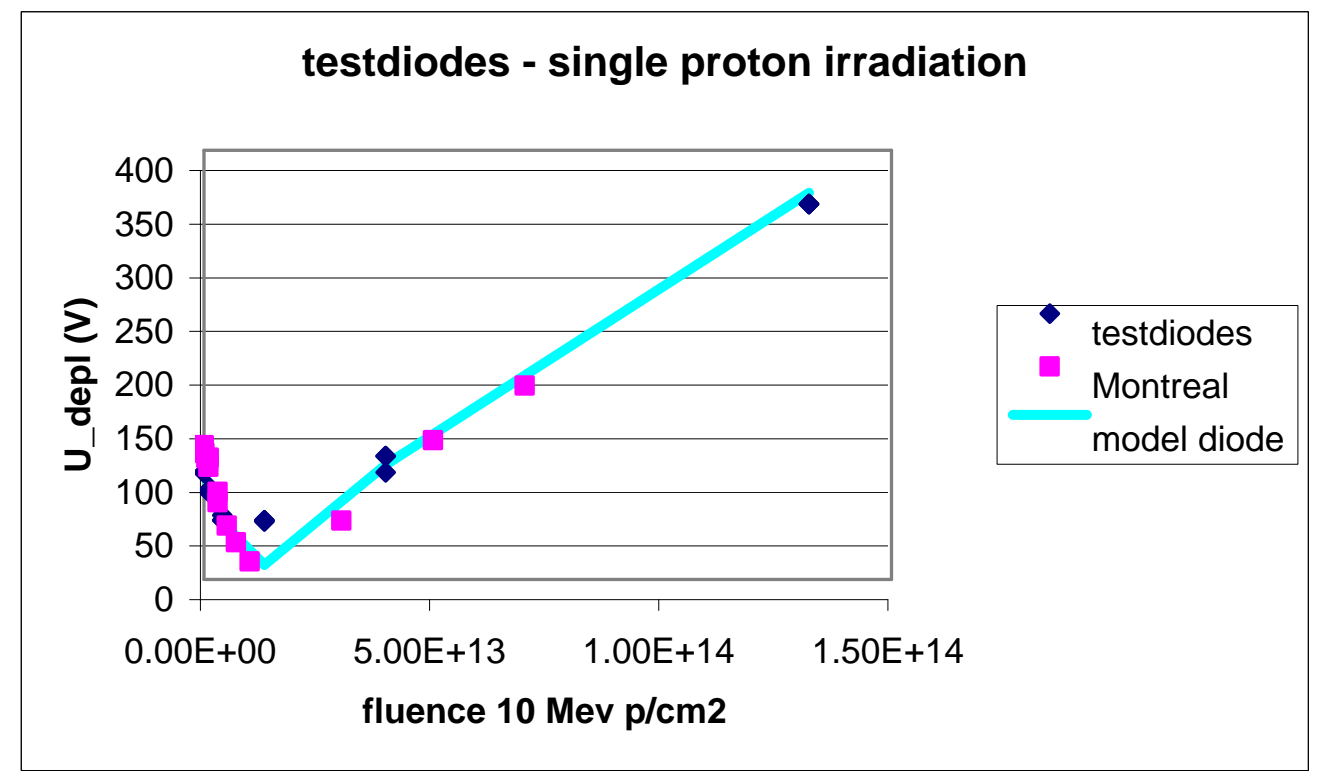

Figure 10: Depletion voltage as function of proton fluence for the testdiodes. The diodes have been irradiated only once. We use the same parameterization model than given in reference 3.

${ }^{11}$ ROSE Collaboration, $3^{\text {rd }}$ RD48 Status report, CERN/LHCC 2000-009 
The depletion voltage values as a function of the exposed $10 \mathrm{MeV}$ proton fluences of the HPK sensors is presented in Figure 11. As opposed to the diodes, the HPK sensors were irradiated in several irradiation sessions and after each proton exposure annealed according to our prescription. Compared to the diode data in Figure 10 the HPK sensors show towards high proton fluences a different depletion voltage dependence, i.e. the effective doping concentration grows stronger with fluence than for the diodes. This is attributed mainly to the multiple annealing steps of the sensors indicating that also reverse annealing especially during the last two to three irradiation sessions took place. In order to compare the obtained depletion voltage changes for those sensors, the multiple annealing together with the temperature and irradiation history has to be taken into account, and a more complex radiation damage parameterization has to be applied. The plot of Figure 11 contains a depletion voltage prediction based on the so-called Hamburg model $^{12}$. This parameterized model describes the decrease of the initial $n$ type impurity with radiation as $\mathrm{N}_{\mathrm{eff}}=\mathrm{N}_{\mathrm{eff}, 0}-\mathrm{N}_{\mathrm{dam}}(\phi, \mathrm{t}, \mathrm{T})$. The introduced damage $\mathrm{N}_{\mathrm{dam}}$ can be classified in three types:

- $\quad$ stable damage: $\mathrm{N}_{\mathrm{c}}=\mathrm{N}_{\mathrm{c} 0}(1-\exp (-\mathrm{c} \phi))+\mathrm{g}_{\mathrm{c}} \cdot \phi$

- $\quad$ short term annealing: $\mathrm{N}_{\mathrm{a}}(\phi, \mathrm{t}, \mathrm{T})=\mathrm{g}_{\mathrm{a}} \cdot \phi \cdot \exp \left(-\mathrm{k}_{\mathrm{a}} \cdot \mathrm{t} \cdot \exp \left(-\mathrm{E}_{\mathrm{aa}} / \mathrm{kT}\right)\right)$

- reverse annealing $\mathrm{N}_{\mathrm{Y}}(\phi, \mathrm{t}, \mathrm{T})=\mathrm{g}_{\mathrm{Y}} \cdot \phi \cdot\left(1-1 /\left(1+\mathrm{k}_{\mathrm{Y}} \cdot \mathrm{t} \cdot \exp \left(-\mathrm{E}_{\mathrm{a}} / \mathrm{kT}\right)\right)\right)$

We calculated the three components after each irradiation step by taking into account the short term and reverse annealing effects. The conversion to the $1 \mathrm{MeV}$ equivalent neutron fluence that enter into these equations, was done by using our best estimate of the beam hardness, which comes from the leakage current determinations. Therefore, the recorded $10 \mathrm{MeV}$ proton fluence values have been scaled by a factor of 2.54 , instead of the theoretically expected scaling factor of 3.87. At the end we found a reasonable agreement of the model to our data for the following set of parameters:

\begin{tabular}{lllllllllll} 
Parameter: & $\boldsymbol{C}$ & $\boldsymbol{g}_{\boldsymbol{c}}$ & $\boldsymbol{g}_{\boldsymbol{a}}$ & $\boldsymbol{k}_{\boldsymbol{a}}$ & $\boldsymbol{E}_{\boldsymbol{a} a}$ & $\boldsymbol{g}_{\boldsymbol{y}}$ & $\boldsymbol{k}_{\boldsymbol{y}}$ & $\boldsymbol{E}_{\boldsymbol{a y}}$ & $\boldsymbol{N}_{\boldsymbol{c} \boldsymbol{0}} / \boldsymbol{N}_{\text {eff;0 }}$ \\
\hline Value: & $1.13 \mathrm{E}-13$ & $1.9 \mathrm{E}-2$ & $1.81 \mathrm{E}-2$ & $2.4 \mathrm{E} 13$ & 1.09 & $6.6 \mathrm{E}-2$ & $1.5 \mathrm{E} 15$ & 1.325 & 1 \\
& & & & & & & & & & \\
& & & & & & & & & & \\
Unit: & $\mathrm{cm}^{-2}$ & $\mathrm{~cm}^{-1}$ & $\mathrm{~cm}^{-2}$ & $\mathrm{~s}^{-1}$ & $\mathrm{eV}$ & $\mathrm{cm}^{-1}$ & $\mathrm{~s}^{-1}$ & $\mathrm{eV}$ & - \\
& & & & & & & & & & \\
\end{tabular}

Table 1: Used parameters in the Hamburg model description.

Again, we assume a complete donor removal - as is usual in proton irradiation. This is indicated in the last parameter of Table 1. The other parameters and hence the curve in Figure 11 use a set of pretty standard values for proton irradiation. Although we are

${ }^{12}$ G. Lindstroem, M.Moll, E.Fretwurst. NIMA 426 (1999) 1, and literature cited therein 
sensitive to the reverse annealing effects from previous irradiation sessions, the stable damage is in accordance to the expectations, when our own derived hardness factor is applied. The depletion voltage on the HPK sensors as we measured it on the highest fluence point of almost $1 \cdot 10^{14} 10 \mathrm{MeV} \mathrm{p} / \mathrm{cm}^{2}$, is around $350 \mathrm{~V}$. This means that we have a considerable safety margin in the biasing of our detectors, since they can be operated up to $700 \mathrm{~V}$ without any sign of a major junction breakdown.

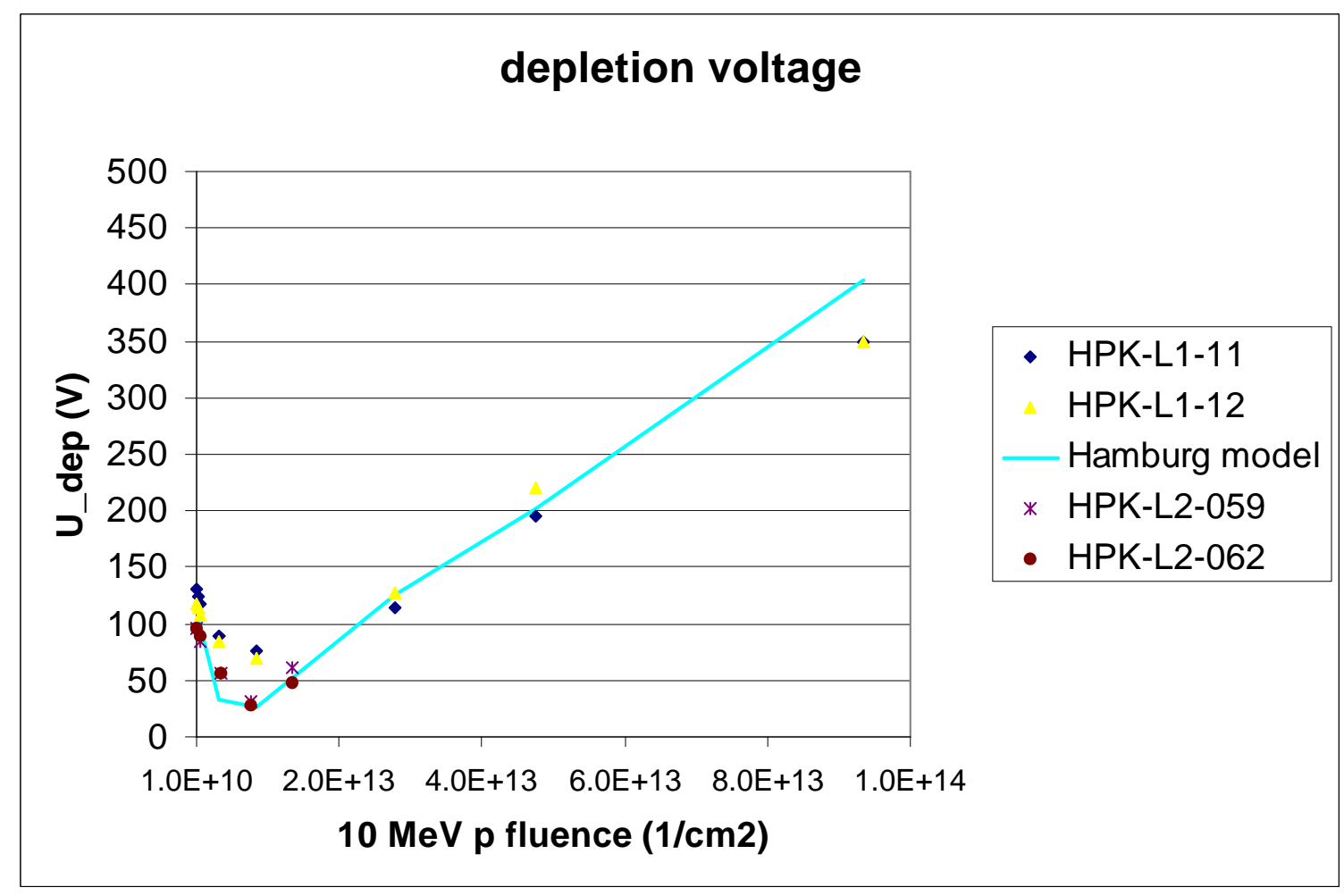

Figure 11: Depletion voltage of the HPK sensors as function of the $10 \mathrm{MeV}$ proton fluence. Shown is a parameterization based on the Hamburg model.

\section{Other electrical measurements on irradiated sensors}

\subsection{Total Load and Interstrip Capacitance on Test Structure}

The total load and interstrip capacitance was measured on various structures as outlined in the Quality Assurance document ${ }^{13}$. This procedure was followed for all our measurements. For ease of comparison, we list here all the load and interstrip capacitance measurements performed. The results on the unirradiated sensors or test structures are also published in the L1 characterization note ${ }^{14}$.

\footnotetext{
${ }^{13}$ http://www.physik.unizh.ch/ lehnerf/dzero/qa/qa.html

${ }^{14}$ M. Demarteau et al., Characteristics of the Layer 1 Silicon Sensors for the Run IIb Silicon Detector
} 
We define the load capacitance as the total capacitance to ground seen by a readout strip. This load capacitance consists of the interstrip capacitance to both neighbor strips and the backplane capacitance. Figure 12 shows the total load capacitance as function of frequency as measured on a baby sensor of the test structure. Figure 13 shows the interstrip capacitance for the same structure. As expected due to the narrow strips compared to the thickness of our detectors, the interstrip capacitance dominates the load capacitance. The backplane capacitance contributes only approximately $30 \%$ to the total.

Total strip capacitance (pF)

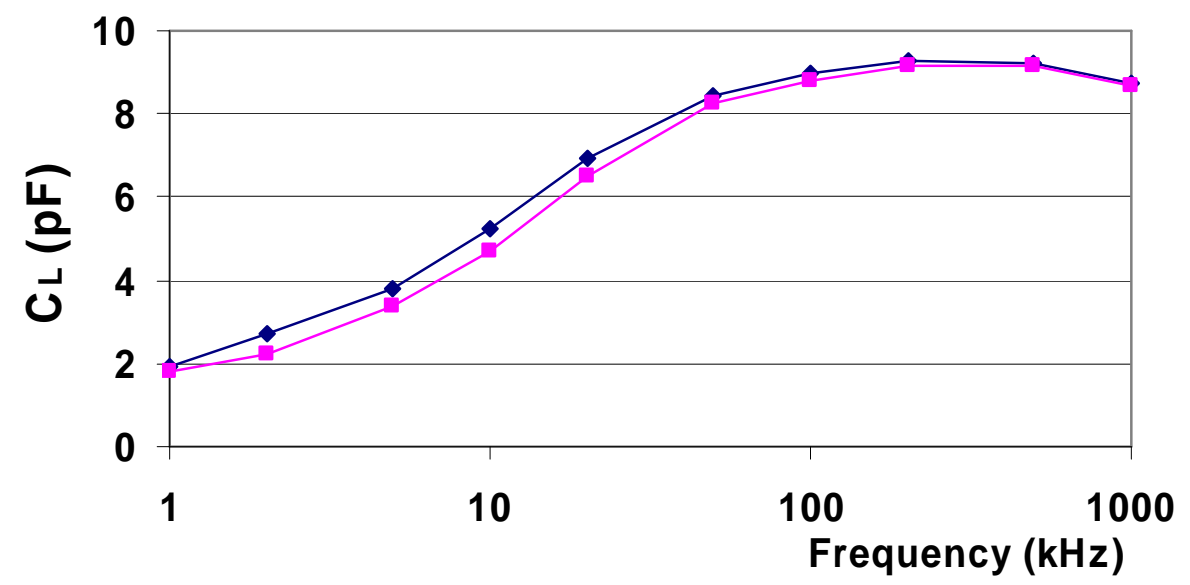

Figure 12: Total load capacitance as function of frequency as measured on the unirradiated baby sensor.

\section{Interstrip capacitance}

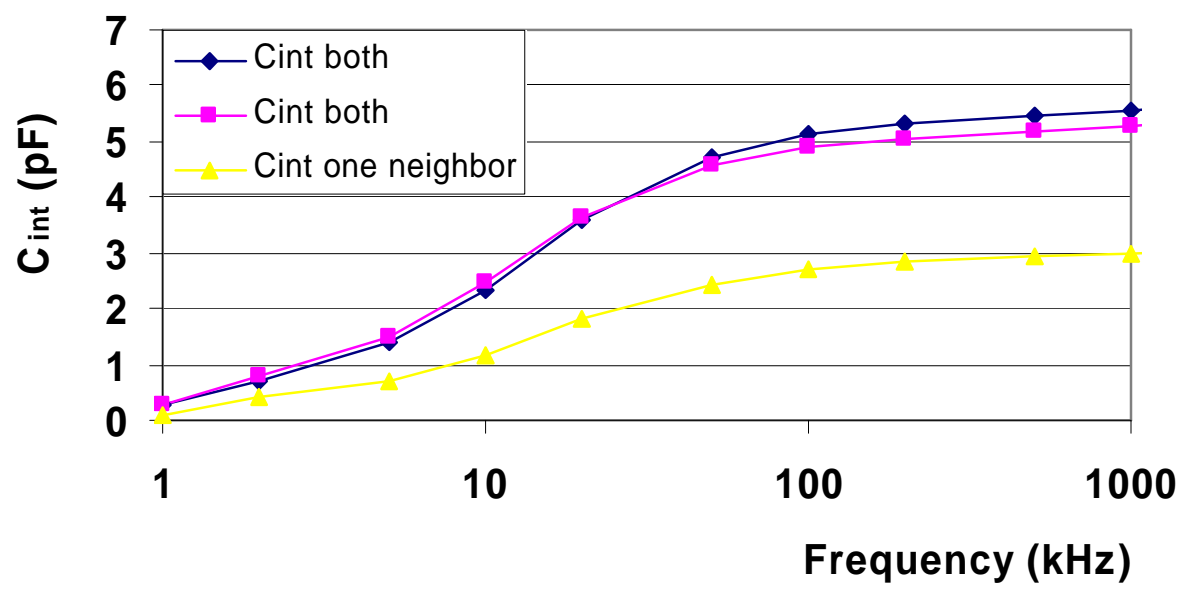

Figure 13: Interstrip capacitance as function of frequency, with respect to one and two neighbors for unirradiated baby sensors. 


\subsection{Total Load and Interstrip Capacitance on Sensors}

Figure 14 shows the dependence of the total load capacitance $\mathrm{C}_{1}$ as function of frequency for the triplet of strips $(193,194,195)$ on L1-sensor 1 . At a frequency of $1 \mathrm{MHz}$, the frequency of relevance for operation with the SVX4 readout chip, the total capacitance is about $1.1 \mathrm{pF} / \mathrm{cm}$. The interstrip capacitance is presented in Figure 15. The measured capacitances on the test structures agree well with the capacitances obtained on the sensors.

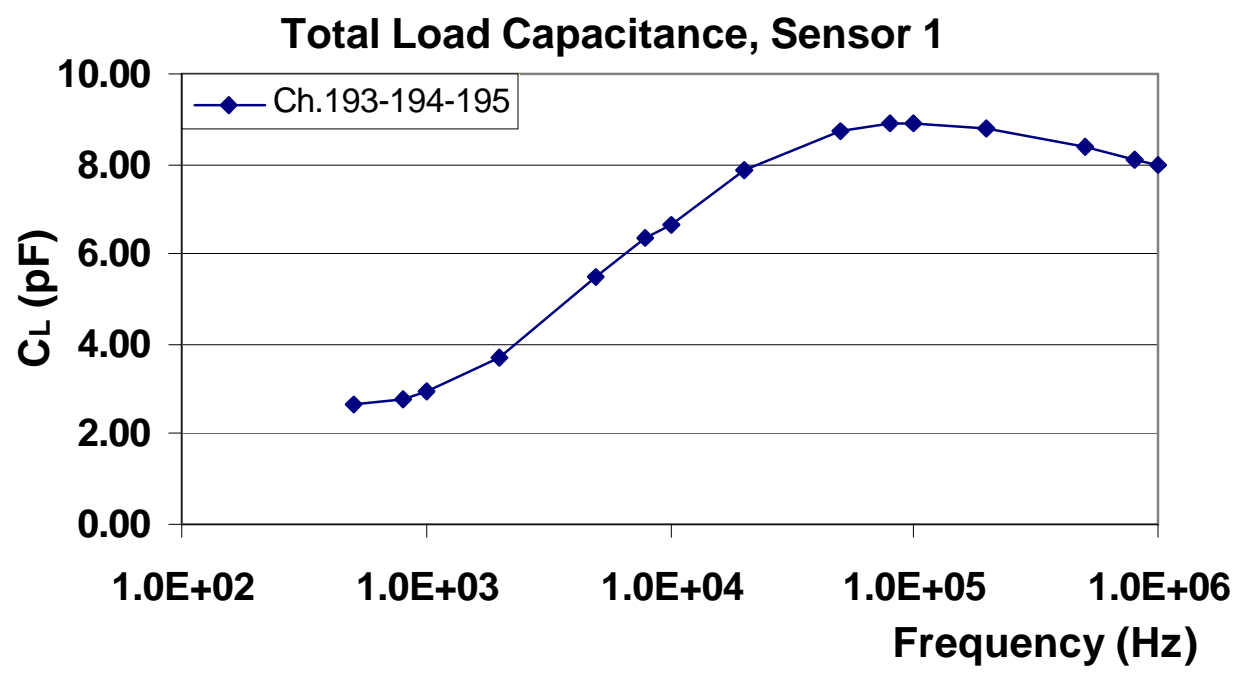

Figure 14: Frequency dependence of total load capacitance for an unirradiated sensor. Note the frequency is in $\mathrm{Hz}$.

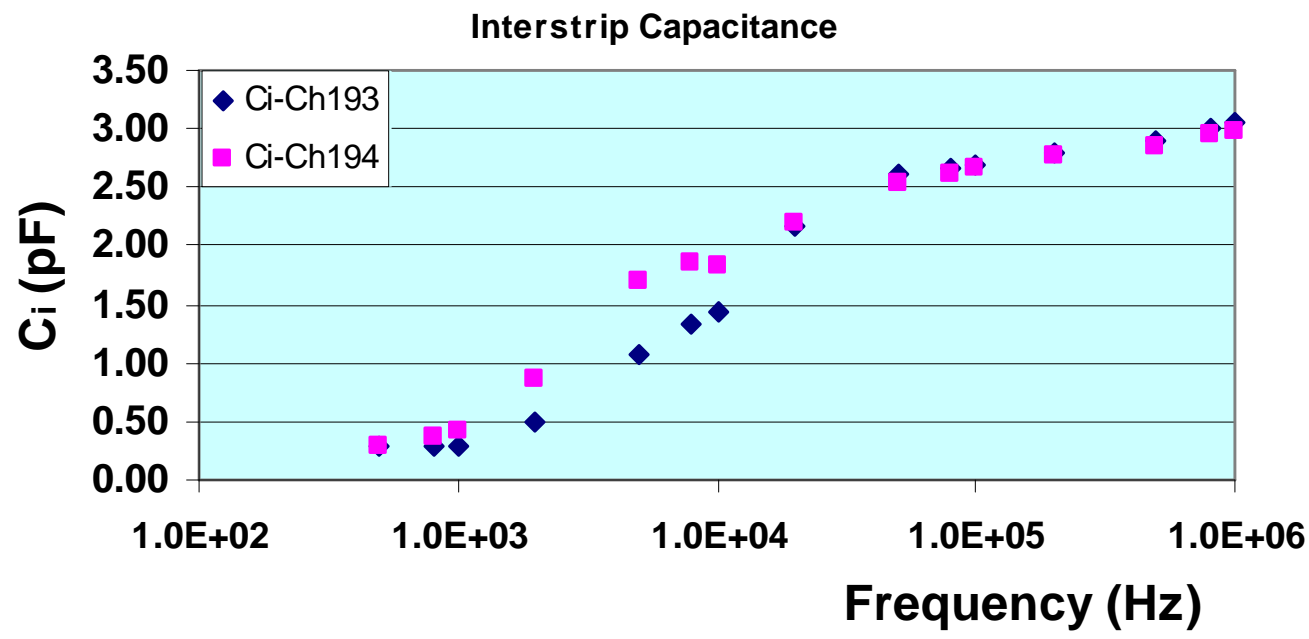


Figure 15: Interstrip capacitance to one neighbor strip as function of frequency $(\mathrm{Hz})$ for two strips of sensor 1 (an unirradiated sensor).

\subsection{Total Load and Interstrip Capacitance on Irradiated Sensor}

The capacitance measurements were also carried out on L1 sensor 11 after irradiation at KSU. The total flux that this sensor received was $9.3510^{13} 10 \mathrm{MeV} \mathrm{p} / \mathrm{cm}^{2}$. Figure 16 and Figure 17 show the load and interstrip capacitance for this sensor, respectively. Note that the interstrip capacitance is defined to one neighbor only and hence shown consistently to the other plots. We observe that after irradiation both capacitance values have increased by almost $40 \%$ at $1 \mathrm{MHz}$. The load capacitance, i.e. the total capacitance for the preamplifier to ground at $1 \mathrm{MHz}$ for the $7.74 \mathrm{~cm}$ long strip in layer 1 is now between 1.4 to $1.5 \mathrm{pF} / \mathrm{cm}$ in contrast to the unirradiated case of a load capacitance of $1.1 \mathrm{pF} / \mathrm{cm}$. This capacitance increase is entirely due to enhanced interstrip capacitances as Figure 17 demonstrates.

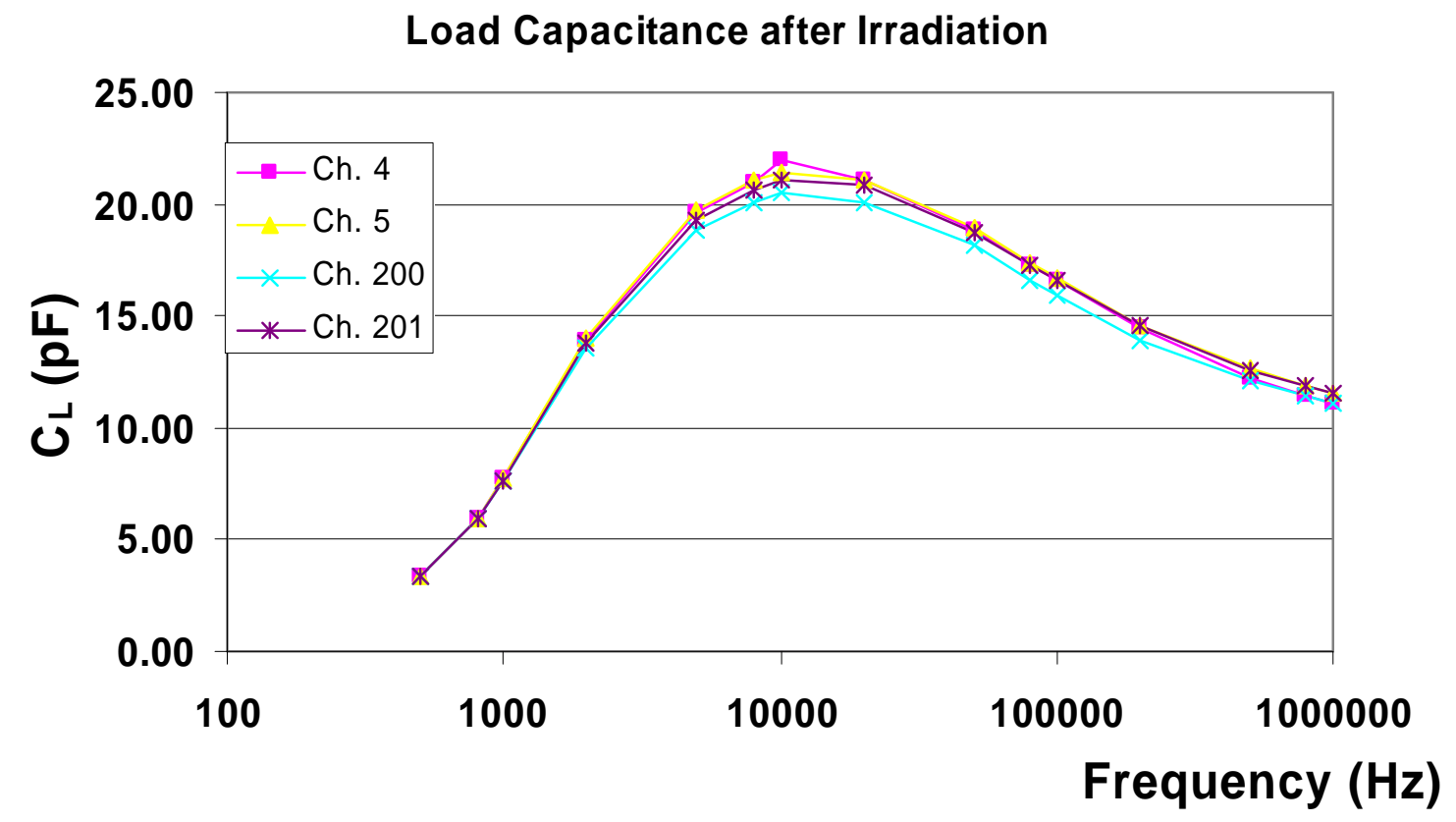

Figure 16: Total load capacitance for Layer 1, sensor 11, after irradiation to $9.35 \cdot 10^{13} 10 \mathrm{MeV} \mathrm{p} / \mathrm{cm}^{2}$. 


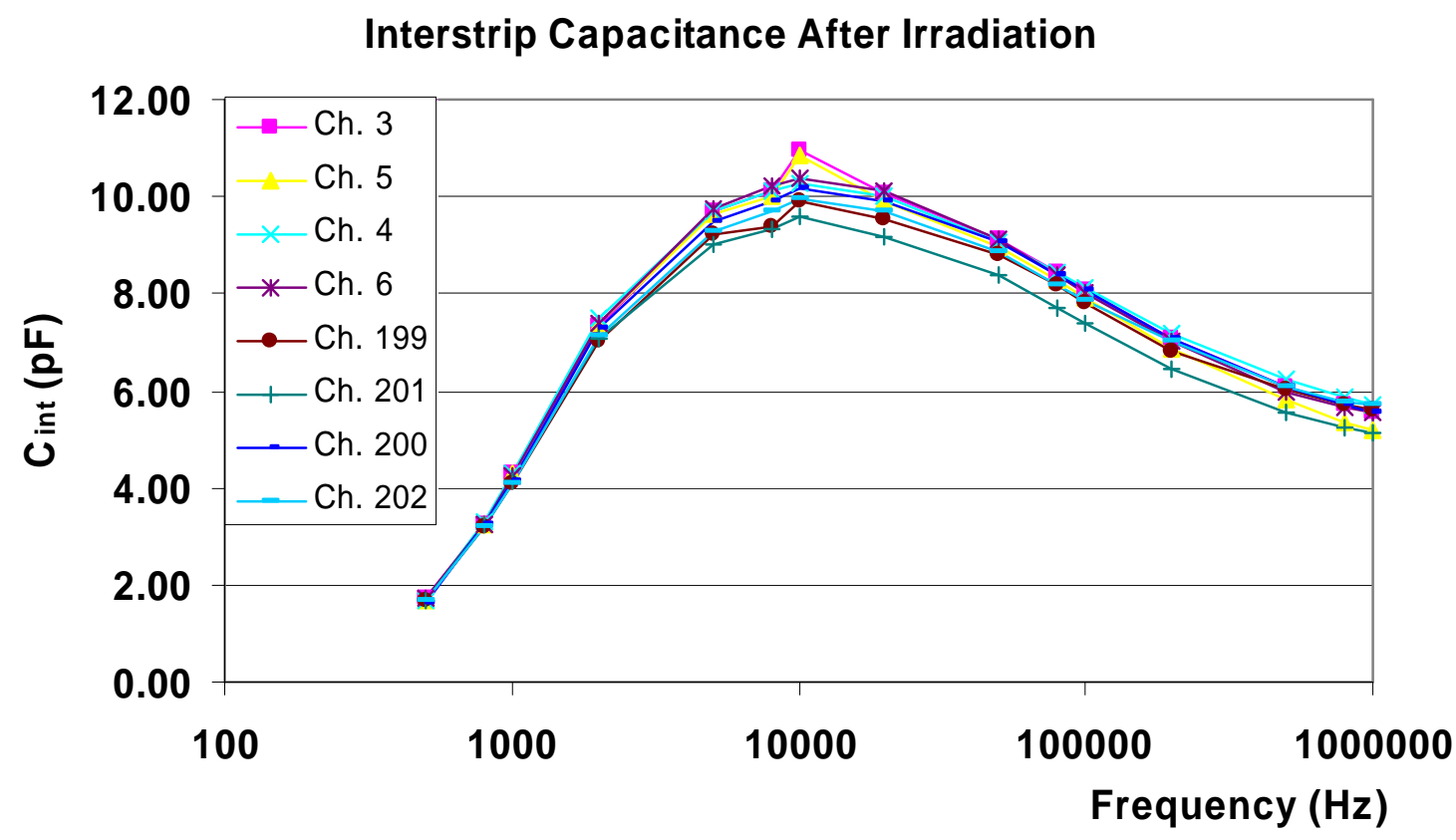

Figure 17: Interstrip capacitance of Layer 1 sensor 11 after irradiation to $9.35 \cdot 10^{13} 10 \mathrm{MeV} \mathrm{p} / \mathrm{cm}^{2}$.

The bias dependence of the load capacitance for this irradiated sensor is shown in Figure 18. The capacitance does not really drop towards higher $\mathrm{HV}$ and reaches a value of around $11 \mathrm{pF}$ for the $7.74 \mathrm{~cm}$ long strip detector, corresponding to $1.42 \mathrm{pF} / \mathrm{cm}$.

We should note that the investigated sensor has received a dose which we expect for layer 0 sensors after an accumulation of $20 \mathrm{fb}^{-1}$ of luminosity. The expected dose number includes a safety factor of 1.5 and our hardness factor for the $10 \mathrm{MeV}$ proton beam of 2.54. The expected increase in ENC noise due to Johnson noise of the capacitive load for a one-sensor ladder in layer 0 including a $45 \mathrm{~cm}$ long Kapton interconnect cable with additional $0.45 \mathrm{pF} / \mathrm{cm}$ capacitance will only be from around $1680 e$ to $1815 e$ and hence will not degrade the $\mathrm{S} / \mathrm{N}$ significantly ${ }^{15}$. Finally, a module test using this irradiated sensor is in preparation. A noise measurement will then clarify by how much the noise due to the larger interstrip capacitance has really increased.

\footnotetext{
${ }^{15}$ Note 1 reports on the noise projections for layer 0 and layer 1 sensors during Run IIb operation. The note assumed a now very realistic load capacitance of $1.5 \mathrm{pF} / \mathrm{cm}$ after irradiation.
} 


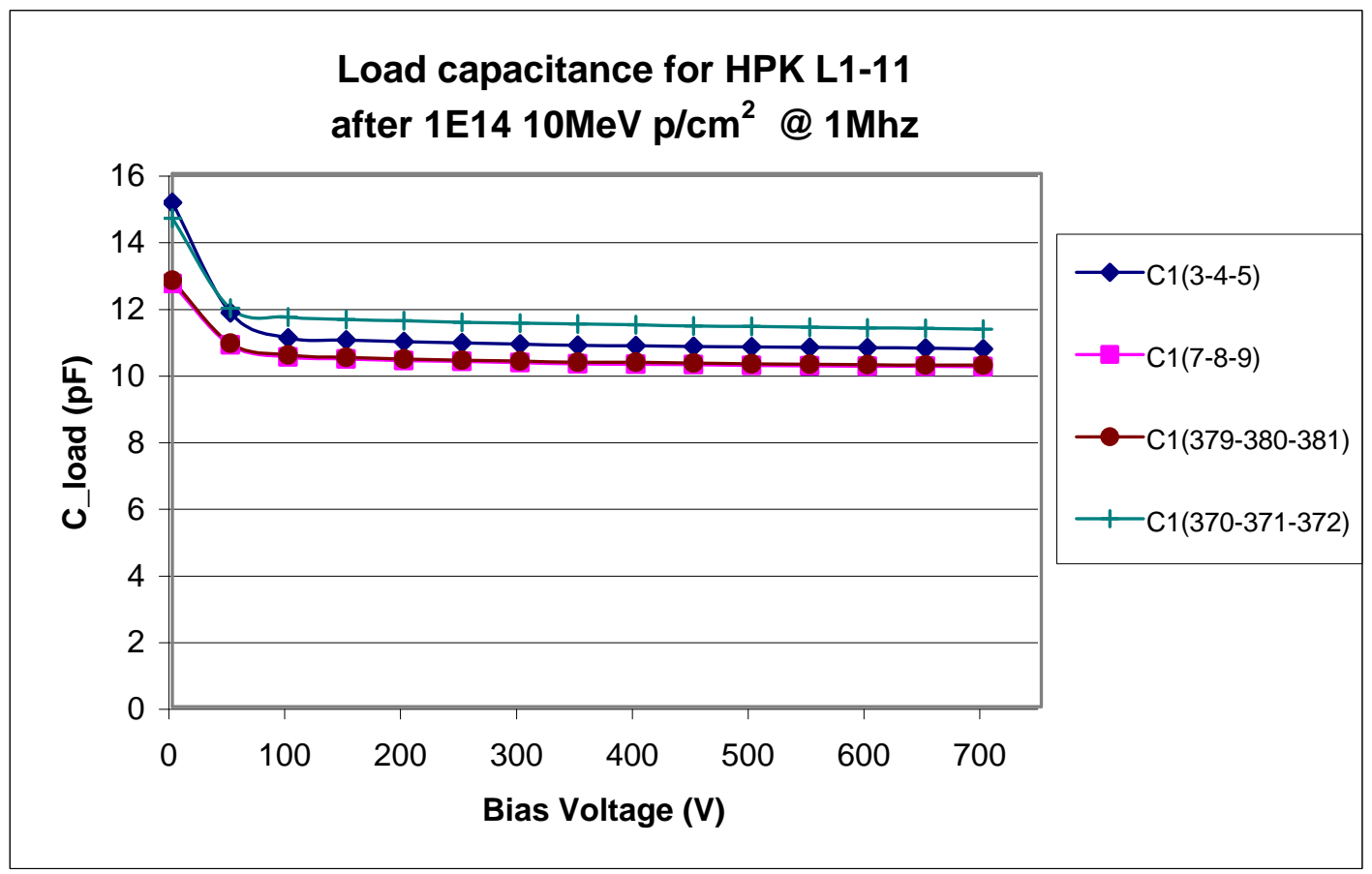

Figure 18: Load capacitance for HPK-L11 after the final fluence point of almost $1 E 1410 \mathrm{MeV} \mathrm{p/ \textrm {cm } ^ { 2 } . ~ T h e ~}$ capacitance has been evaluated at $1 \mathrm{MHz}$. 


\subsection{Resistance Measurements}

We have measured the resistance of the poly-silicon resistor and the combined resistance of the poly-silicon resistor and implant resistance on one irradiated L1 sensor. Figure 19 shows the I-V curve for the determination of the poly-silicon resistor value. A resistance of $0.63 \mathrm{M} \Omega$ was measured. The measurement was repeated for several strips and all measurements are in good agreement.

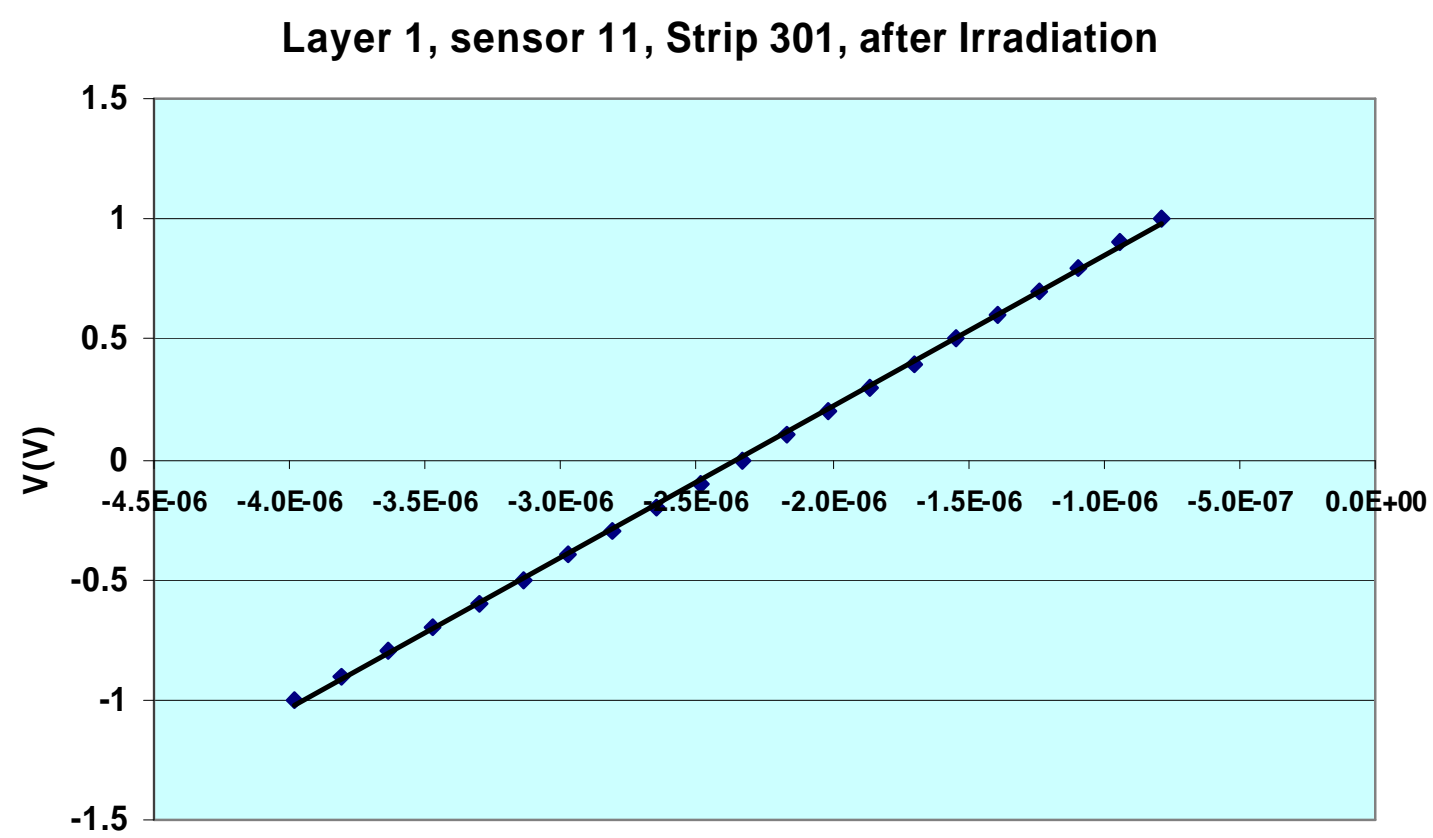

I(A)

Figure 19: I-V curve for determination of poly-silicon resistor value on sensor 11 after irradiation.

Figure 20 shows the measurement of the total implant plus poly-silicon resistance. A resistance of $1.5 \mathrm{M} \Omega$ is measured. Also here, measurements on other strips yield similar results. All the resistance measurements are in close agreement with the measurements on the non-irradiated sensors that are published in note 14 . It can therefore safely be concluded that irradiation does not change the resistance. 


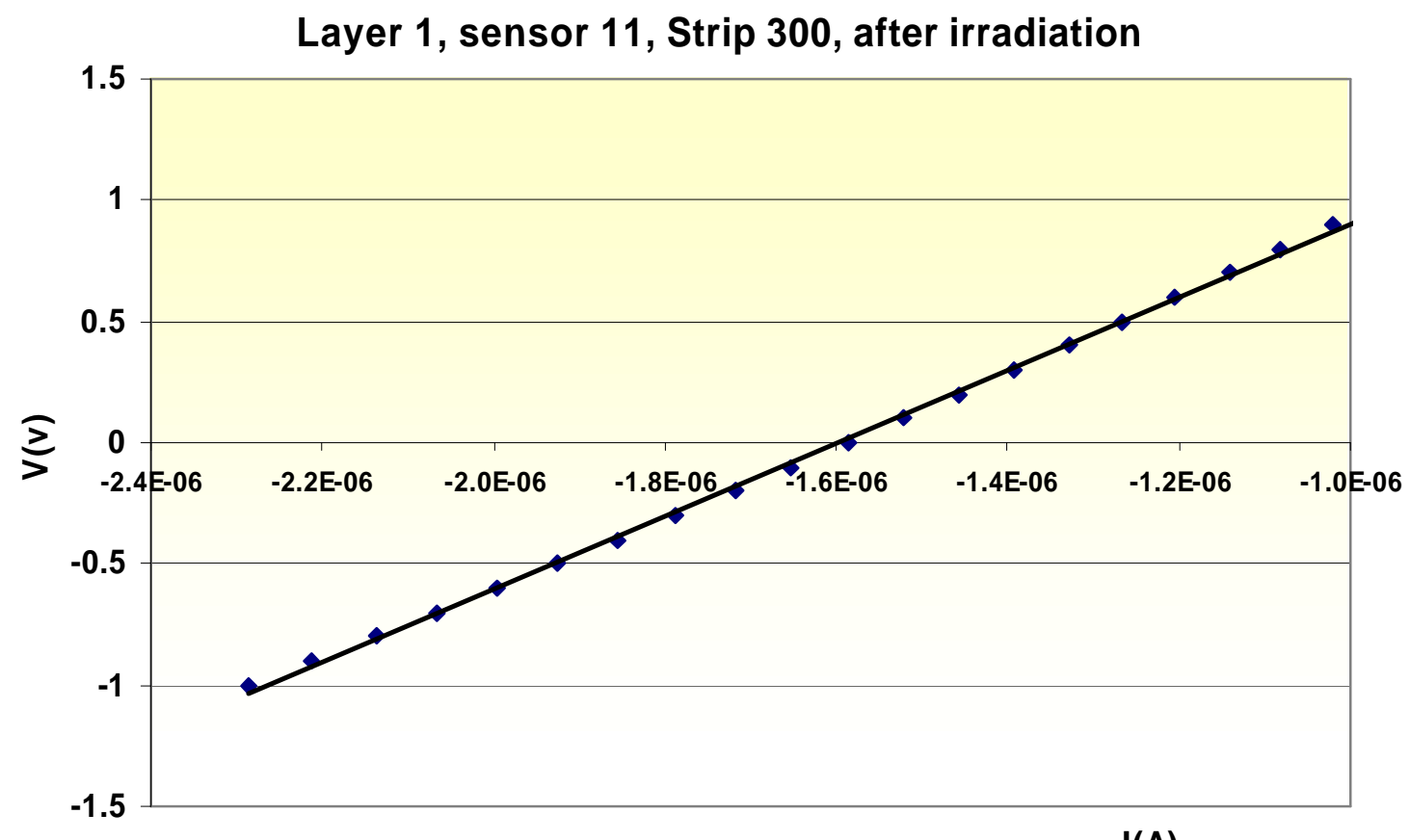

$\mathrm{I}(\mathrm{A})$

Figure 20: I-V curve for the determination of the total implant and poly-silicon resistance for strip 300 of Layer 1 sensor 11 after irradiation

\section{Conclusions}

We have performed an irradiation study on HPK layer 1 silicon sensors and planar diodes with $10 \mathrm{MeV}$ protons at the JR Macdonald Laboratory of KSU. The sensors were irradiated in multiple steps and exposed to a maximum proton fluence of up to $10^{14} 10$ $\mathrm{MeV} \mathrm{p} / \mathrm{cm}^{2}$. The diodes were irradiated in single shots up to a fluence of $1.32 \cdot 10^{14} 10$ $\mathrm{MeV} \mathrm{p} / \mathrm{cm}^{2}$. The annealing scheme of $80 \mathrm{~min}$ at $60 \mathrm{C}$ after each irradiation step was followed. The proton flux calibration was carefully crosschecked by a copper activation analysis method and confirmed to be consistent within $20 \%$.

The results on increasing leakage currents towards larger fluences show, that the observed bulk radiation damage is $30-40 \%$ less than what the simple application of the NIEL scaling hypothesis would predict. The hardness factor for the $10 \mathrm{MeV} p$ beam is extracted from our leakage current results and is determined to be 2.54 . The theoretical NIEL scaling yields 3.87 for that proton energy if the silicon sensors are assumed to have negligible thickness. Thick silicon sensors of $300 \mu \mathrm{m}$ are expected to yield hardness values of 4.5 for the NIEL scaling hypothesis.

Our leakage current results are in very good agreement to a previous irradiation study at $10 \mathrm{MeV}$ protons, confirming the breaking of the NIEL hypothesis at low proton energies. The hardness factor for $10 \mathrm{MeV}$ protons, which is found in our study, can be used to convert to an equivalent $1 \mathrm{MeV}$ neutron fluence. This means that the full radiation dose 
of $10^{14} \mathrm{p} / \mathrm{cm}^{2}$ would then correspond to roughly $2.5 \cdot 10^{14} 1 \mathrm{MeV} \mathrm{n} / \mathrm{cm}^{2}$ or almost $20 \mathrm{fb}^{-1}$ of accumulated luminosity of Run IIb in the innermost layer. After receiving such a large dose, we do not see any evidence for a clear junction breakdown on our sensors. The depletion voltage evolution of the diodes towards higher fluences can be described by a simple parameterization assuming full donor removal as is known in proton irradiation. The more complex Hamburg model was used to describe the depletion voltage behavior of the HPK L1 sensors since they have been annealed several times. The measurements show that the sensors deplete at around 350-400V at the highest fluence points. Since we can bias them up to $700 \mathrm{~V}$ without major problems, we still have enough safety margins for the operation.

We have measured the load and interstrip capacitances on an L1 sensor, that was irradiated up to almost $1 \cdot 10^{14} 10 \mathrm{MeV} \mathrm{p} / \mathrm{cm}^{2}$. We observed that the load capacitance at 1 $\mathrm{MHz}$, which is the relevant frequency for the SVX-IV chip operation, increases by almost $40 \%$ compared to unirradiated sensors, i.e. from $1.1 \mathrm{pF} / \mathrm{cm}$ to up to $1.5 \mathrm{pF} / \mathrm{cm}$. This increase is due to enhanced interstrip capacitances and will additionally lead to a small increase in preamplifer noise beside shot noise after irradiation. The load capacitance showed no significant HV dependence beyond the depletion voltage. Further measurements demonstrate that there is no change in the resistances, neither for the polysilicon resistors nor for the implant resistance after irradiation. 